\title{
Testing Traffic Control Strategies in Gaborone Using Dynamic Traffic Assignment Tools
}

\author{
Eleni Papatzikou*, Antony Stathopoulos** and Panos Papadakos*** \\ * NAMA Consulting Engineers and Planners S.A., 32 Perrikou St, 11524, Athens, Greece, (e-mail: epapatzikou@namanet.gr) \\ ** Laboratory of Railways and Transport, School of Civil Engineering, National Technical University of Athens, 5 Iroon \\ Polytechniou, 15773 Athens, Greece, (e-mail: a.stath@transport.ntua.gr) \\ *** NAMA Consulting Engineers and Planners S.A., 32 Perrikou St, 11524, Athens, Greece, (e-mail: ppapada@namanet.gr)\}
}

\begin{abstract}
Dynamic Traffic Assignment (DTA) models are widely used for online and offline applications for efficient deployment of traffic control strategies and the evaluation of traffic management schemes and policies. A valid statistical methodology is proposed for assessing the successful implementation of traffic control strategies with regard to their inherit risk at the network efficiency and therefore providing a useful and easily applicable tool for practitioners in selecting the less risky alternative. In this paper we have considered a common method, Value-at-Risk assessment of financial portfolios, for the assessment of traffic control strategies using Dynamic Assignment Tools in the City Centre of Gaborone in Botswana and the results are presented here.
\end{abstract}

\section{INTRODUCTION}

The evolution of Intelligence Transportation Systems (ITS) and Advanced Traveler Information Systems (ATIS) has led to the increasing use of Dynamic Traffic Assignment (DTA) models in a variety of on-line, off-line applications and incorporated in transportation planning studies $(T R B, 2011)$. DTA modelling, introduced by Yagar (1971), Robillard (1974), and Merchant and Newhauser (1978) among others, is the temporal extension and disaggregation of the classical static assignment problem, which assumes steady traffic conditions over the study period, in order to better capture realistic traffic conditions. Therefore, the aim of a DTA model is to determine the network flow patterns given an Origin-Destination (O-D) demand, a network structure and link performance functions incorporating time dependencies in both the supply and the demand attributes (Peeta and Yang, 2003). An extensive review of the DTA models and the formulation, characteristics, solution methodologies, limitations and practical applications is provided in Peeta and Ziliaskopoulos (2001), Boyce et al. (2001), TRB (2011) and FHWA (2012).

The implementation of a DTA model on a large-scale network causes important challenges to the modeling procedure, such as the manipulation of network and demand data, the modeling of turning movements, the efficient computation of link travel times and the handling of complex path data (Ziliaskopoulos et al., 2004). The evaluation, development and optimization of Intelligent Transport Systems with regard to the successful deployment of traffic control strategies presume stable and robust network states. The development, application and optimization of control strategies and operation policies are based on the estimation of time dependent traffic states which depict the network efficiency through time evolution aiming to its enhancement.
The efficiency of a network is commonly calculated and evaluated in three levels of detail, system-wide analysis, corridor analysis and, in more detail, node analysis. Network and corridor efficiency is generally associated with measurements and representations of network speed, distance travelled, travel time, generalized cost etc. Node analysis with regard to efficiency is generally associated with estimation of delays, queues, level of service, etc.

The necessity to restrict and minimize the time dependent system risks and vulnerabilities stimulates the need for valid statistical analyses of the network performance and representation of traffic conditions. Risk is generally defined as the potential loss resulting from certain conditions. Risk models have been extensively used in many research fields to identify and evaluate the risk of certain conditions based on a given threshold over a modelling horizon. In particular, Value-at-Risk (VaR) model has been widely used in financial applications. Over the past years, VaR has also been used in many engineering research fields, such as transportation of hazardous materials (Toumazis et.al., 2013), network resilience (Cheliotis and Kenyon, 2002, Mastroeni and Naldi, 2011), network design and location problems (Sun and Gao, 2012, Wang et al., 2009), water management (Shao et al., 2011) and many others. In the framework of estimating the dynamic traffic conditions with DTA models, we define risk as the potential loss of budgeted travel time based on the selected network and control strategy, demand distribution and parameters setup over the simulation horizon.

In this paper we focus on formulating a methodology for testing different control strategies using dynamic traffic assignment tools applied at the City Centre of Gaborone (Botswana). Our objective is to assess the risk to the network efficiency resulting from deployment of the proposed traffic control strategies by using a Value-at-Risk model. The proposed methodology can be easily implemented by researchers or practitioners to evaluate their alternative 
strategies and aid them to choose the alternative with the less potential risk. The paper is organized as follows: the outline of the proposed methodology is presented in section 2 , the risk analysis is presented in section 3, the application at the City Centre of Gaborone (Botswana) and the network and model setup are described in section 4, the results are presented in section 5 and the main conclusions are summarized in section 6 .

\section{METHODOLOGY}

The proposed methodology is an iterative process for the assessment of traffic control strategies using dynamic assignment tools. Based on the Dynamic Traffic Assignment, we calculate the performance indices for each simulation (time) step. Using a Value-at-Risk analysis, we identify the critical values of performance indices for the corresponding simulation step. If the critical values are within acceptable bounds, the iterative process stops, otherwise a new control strategy is developed and deployed to the network and once again the prevailing traffic conditions are simulated using the DTA model.

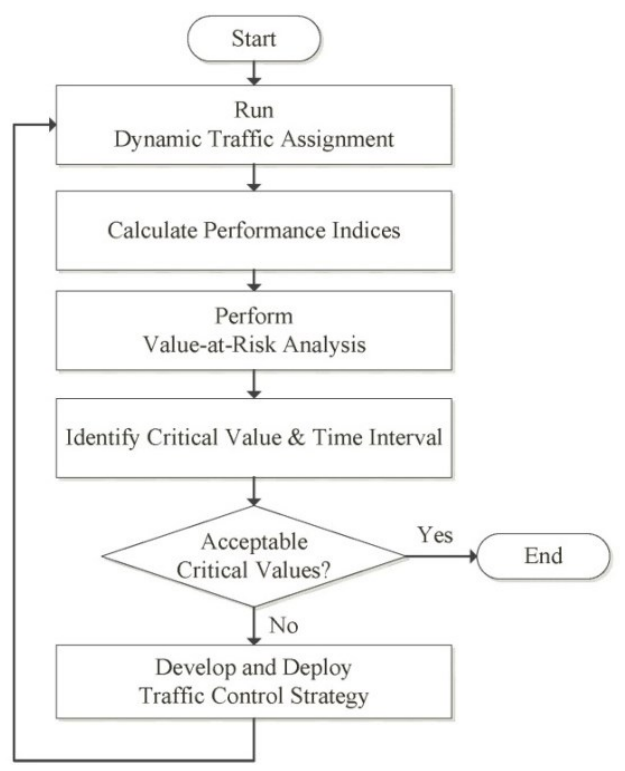

Fig. 1. Methodology for evaluating traffic control strategies

\section{RISK ANALYSIS}

The Value-at-Risk, VaR, model as mentioned above was implemented in Toumazis et al. (2012). This paper presents an adjusted application of this model. The $V a R$ of a network loading solution $l$ is defined as the critical value $\beta$ such that the value of the risk measure $R$ exceeding that value $\beta$ has probability less than or equal to $(1-a)$, where $a$ is the confidence level (i.e. 90\%).

$$
V a R_{a}^{l}=\min \left\{\beta: \operatorname{Pr}\left(R^{l}>\beta\right) \leq 1-a\right\}
$$

The objective of our problem, given a set of alternative DTA network loading solutions, $L$, representing different traffic control strategies, is to determine the solution, $l \in L$, which has the minimum $\operatorname{VaR}$ :

$$
\operatorname{VaR}_{a}^{*}=\min \left\{\operatorname{VaR}_{a}^{l}: l \in L\right\}
$$

In this paper, we have performed the $V a R$ analysis for each signalized node, defining for each node the critical value $\beta$ that has probability less than or equal to $(1-a)$, as it is defined in Eq.1. The risk measure $R$ for the risk analysis has two attributes: the consequence, $c_{\tau_{\mathrm{j}}}$, which includes travel time losses at each simulation step and the probability of that consequence to happen at each simulation step, $p_{\tau_{\mathrm{j}}}$.

Consider a network represented by a connected graph $G\{A, N\}$, where $A$ are the set of links and $N$ are the set of nodes $(n \in N)$. Each DTA network loading solution, $l$, consists of a sequence of simulation steps, $\tau_{\mathrm{j}}$, disaggregating the entire simulation horizon, $T \quad\left(T=\sum \tau_{\mathrm{j}}\right)$. The consequence for each node analysis is taken as the average delay which is calculated based on the Highway Capacity Manual, HCM2010, (Transportation Research Board, 2010) methodology for each simulation step. The probability, $p_{n, \tau_{\mathrm{j}}}^{l}$ of that consequence to happen is taken for each node as the ratio of the through traffic at each simulation step to the through traffic of that node over the simulation horizon. The consequence, $c_{n, \tau_{\mathrm{j}}}^{l}$, for each node, $n$, per step, $\tau_{\mathrm{j}}$, is the average delay for all approaches during that simulation step, which are sorted in ascending order by the resulting consequence (i.e. the average delay). In the sorted set of the average delay for each node, let $C_{n,(k)}^{l}$ be the $k$-th smallest value in the set of simulation steps $\left\{c_{n, \tau_{\mathrm{j}}}^{l}: \tau_{\mathrm{j}} \in T\right\}$ and $p_{n,(k)}^{l}$ the corresponding probability of that consequence happening. The risk measure $R_{n}^{l}$ takes the following values:

$R_{n}^{l}=\left\{\begin{array}{cccc}0 & \text { with } & \text { probability } & 0 \\ C_{n,(1)}^{l} & \text { with } & \text { probability } & p_{n,(1)}^{l} \\ \vdots & \vdots & \vdots & \vdots \\ C_{n,\left(m_{l}\right)}^{l} & \text { with } & \text { probability } & p_{n,\left(m_{l}\right)}^{l}\end{array}\right.$

where $m_{1}=T / \tau_{\mathrm{j}}$ is the number of the simulation steps of the DTA loading procedure.

The cumulative distribution function, $F_{R_{n}^{l}}(\beta)=\operatorname{Pr}\left(R_{n}^{l} \leq \beta\right)$, of the risk measure is, if $p_{n,(k)}^{l}=\operatorname{Pr}\left(R_{n}^{l}=C_{n,(k)}^{l}\right)$ :

$F_{R_{n}^{l}}(\beta)=\left\{\begin{array}{ccc}1-\sum_{i-1}^{m_{l}} p_{n,(i)}^{l} & \text { if } & \beta \leq 0 \\ \vdots & \vdots & \vdots \\ 1-\sum_{i-k+1}^{m_{l}} p_{n,(i)}^{l} & \text { if } & C_{n,(k-1)}^{l}<\beta \leq C_{n,(k)}^{l} \\ \vdots & \vdots & \vdots \\ 1 & \text { if } & C_{n,\left(m_{l}\right)}^{l} \leq \beta\end{array}\right.$ 
Given that $\operatorname{Pr}\left(R_{n}^{l} \leq \operatorname{VaR}_{n}^{l}\right)>a$ and the above CDF, the $\operatorname{VaR}$ for the solution $l$ at the confidence level $a$ is:

$$
V a R_{n, a}^{l}=\left\{\begin{array}{ccc}
0 & \text { if } & 0<a \leq 1-\sum_{i-1}^{m_{l}} p_{n,(i)}^{l} \\
\vdots & \vdots & \vdots \\
C_{n,(k)}^{l} & \text { if } & 1-\sum_{i-k}^{m_{l}} p_{n,(i)}^{l}<a \leq 1-\sum_{i-k+1}^{m_{l}} p_{n,(i)}^{l} \\
\vdots & \vdots & \vdots \\
C_{n,\left(m_{l}\right)}^{l} & \text { if } & 1-p_{n,\left(m_{l}\right)}^{l}<a \leq 1
\end{array}\right.
$$

Consequently $\beta_{n, a}^{l}=C_{n,(k)}^{l}$ if and only if

$$
\sum_{i-k}^{m_{l}} p_{n,(i)}^{l}<1-a \leq \sum_{i-k+1}^{m_{l}} p_{n,(i)}^{l}
$$

where: $\quad \sum_{i=k+1}^{m_{l}} p_{n,(i)}^{l}=\sum_{i=k+1}^{m_{l}} \operatorname{Pr}\left(R_{n}^{l}=C_{n,(i)}^{l}\right)=\sum_{\tau_{\mathrm{j}} \in T, c_{\tau_{\mathrm{j}}}^{l}>C_{n,(k)}^{l}} p_{n, \tau_{\mathrm{j}}}^{l}$ and $\sum_{i=k}^{m_{l}} p_{n,(i)}^{l}=\sum_{i=k}^{m_{l}} \operatorname{Pr}\left(R_{n}^{l}=C_{n,(i)}^{l}\right)=\sum_{\tau_{\mathrm{j}} \in T, c_{\tau_{\mathrm{j}}}^{l}>C_{(k)}^{l}} p_{n, \tau_{\mathrm{j}}}^{l}$

Therefore, $\beta_{n, a}^{l}$ is the $V a R$ for the solution $l$ for the node $n$ as in Eq. 1 if and only if the following conditions are met:

$$
\sum_{\tau_{\mathrm{j}} \in T, c_{n, \tau_{\mathrm{j}}}^{l}>\beta_{\mathrm{n}, \alpha}^{l}} p_{n, \tau_{\mathrm{j}}}^{l} \leq 1-\alpha<\sum_{\tau_{\mathrm{j}} \in T, c_{n, \tau_{\mathrm{j}}}^{l} \geq \beta_{n, \alpha}^{l}} p_{n, \tau_{\mathrm{j}}}^{l}
$$

The VaR for all signalized nodes is then analyzed regarding their critical value and the critical simulation step in which they occur. These critical values and corresponding critical simulation steps represent the associated risk of the given DTA solution with a certain Traffic Control Strategy. The less risky Traffic Control Strategy (Eq.2) is defined as the DTA solution with the minimum $V a R$ at the majority of signalized nodes.

\section{APPLICATION}

The methodology has been applied to the network of Central Gaborone, Botswana. Gaborone is a fast growing city with significant travel demand growth over the last decade. We used a mesoscopic DTA procedure executed by the DTALite simulation-assignment open-source model. DTALite is a freeware dynamic traffic assignment simulation model developed independently by a subcontractor of SHRP 2 Transport Research Board research program (Understanding the contribution of Operations, Technology, and Design to Meeting Highway Capacity Needs) and released under a GNU General Public License in January 2010.

The computational experiments performed with dynamic user equilibrium algorithm and the spatial queue model as incorporated in DTALite. The size of the simulation step in the DTA procedure plays a significant role on the accuracy of the results and the computational time, which is crucial for online applications. The size of simulation steps in DTA applications typically vary between 5 -min and 20-min. Our dynamic network loading solution had simulation and trip departure step sizes of 15 minutes, constrained by the time resolution of the available demand data.

\subsection{The Gaborone City Centre}

The Gaborone City Centre network consists of 91 nodes, 184 links (on-way direction) and 42 O-D zones. The link and demand parameters were based on the data acquired by the Department of Roads, Ministry of Transport and Communications of the Republic of Botswana. The static demand data $(22,770$ trips $)$ which represents the peak hour between 07:00 and 08:00 was expanded to a 3-hour peak period, 06:00-09:00, (73.452 trips) and dynamically distributed through the 3-hour simulation horizon based on the demand distribution described in the following section.

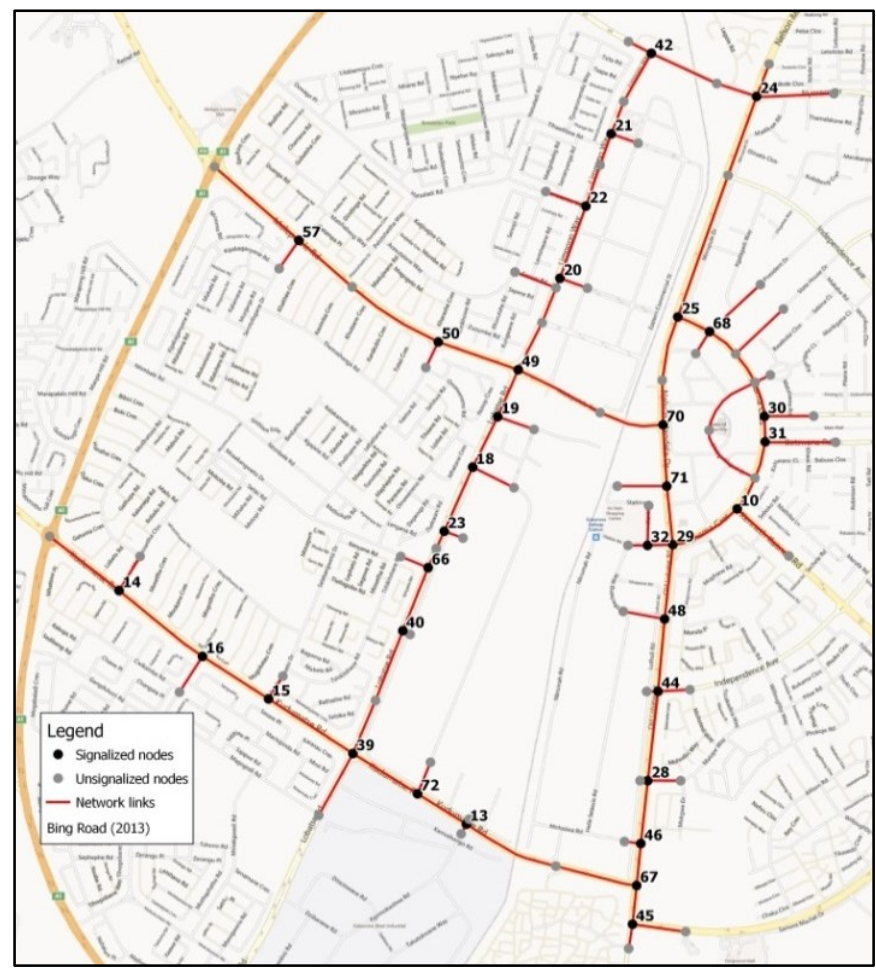

Fig. 2. Simulated network of Gaborone City Centre

\subsection{Demand Distribution}

The resolution of the required time dependent trip matrix used as an input in the DTA procedure depends on the desired modeling accuracy and/ or the variability of the available data. There is extensive research in the literature (e.g. Chang and Tao, 1999; Tsekeris and Stathopoulos, 2003; Stathopoulos and Tsekeris, 2004) regarding the estimation of dynamic O-D trip matrices from observed link flows. A common method for disaggregating the total O-D matrix into finer time resolutions is to use factors derived from the temporal profile of the demand $(T R B, 2011)$. This method was used in this paper for deriving the time dependent O-D matrix from the static peak hour matrix, which is based on actual data. The temporal profile of the demand was derived from continuous traffic counts at 12 cordon points which are entry/ exit points of the study area (Fig.3). The automated cordon counts were totalized every $15 \mathrm{~min}$. 


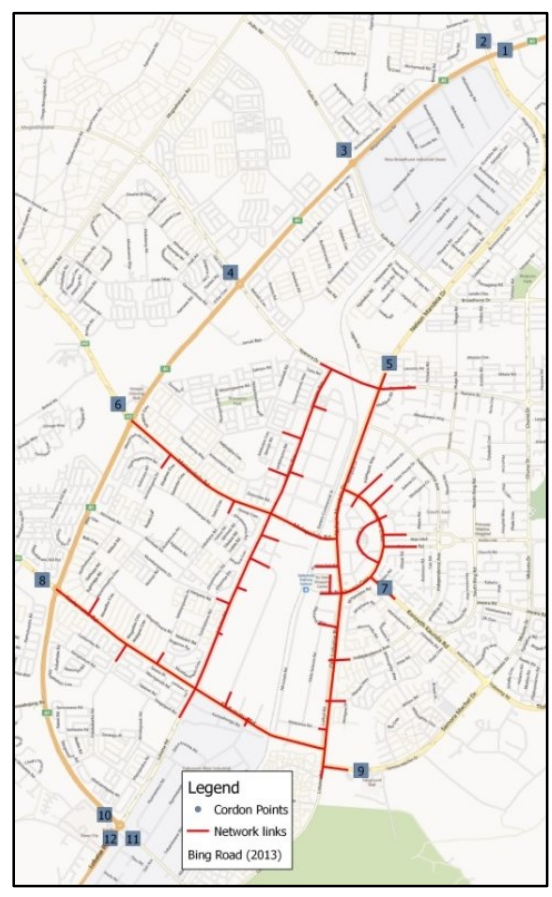

Fig. 3. Cordon points

The resulting loading curve for the demand distribution is presented in Fig.4, where vehicles are assigned following a 15-min loading rate.

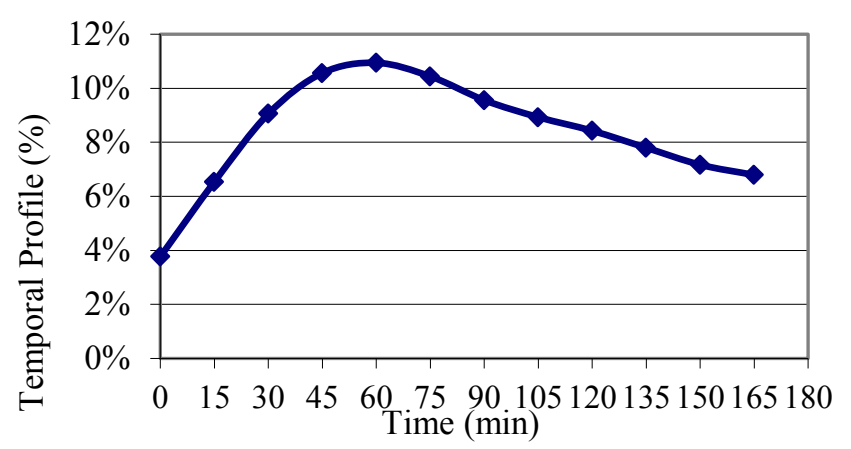

Fig. 4. Demand distribution

\section{RESULTS}

The average delay of all the signalized nodes was calculated based on the HCM 2010 methodology for each simulation step for the baseline conditions. Network statistics for the are presented in Fig.5, where the average speed and the average trip time is calculated for each simulation step. Verification of the results performed for the baseline conditions, limited to simulation test runs.

The $V a R$ analysis was performed with a confidence level $a=90 \%$ and resulted in identifying for each signalized node the critical simulation step and the critical delay that has probability less or equal to $(1-a)$ to occur.

In Fig.6, the histogram of the critical simulation steps for the baseline conditions is presented. It is apparent that most of the signalized nodes have their critical VaR during the 6th and the 7 th simulation step $(07: 15-07: 45)$. Thus, we identify two critical time periods between $07: 15$ and $07: 45$, with regard to the delay.

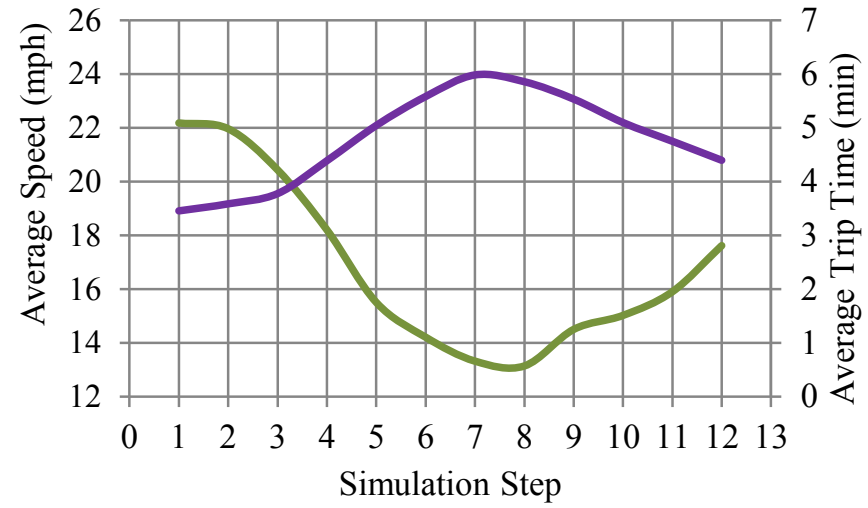

$\longrightarrow$ Average Speed $\longrightarrow$ Average Trip Time

Fig. 5. Network statistics, Baseline

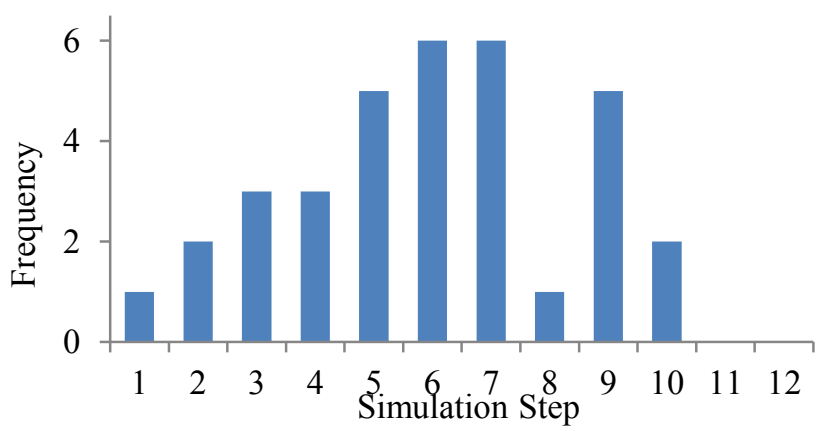

Fig. 6. Histogram of critical simulation step based on VaR analysis, Baseline

Another interesting finding of the attributes of the network refers to the comparison between the Critical Delay with regard to the VaR analysis and the Critical Delay with regard to the Maximum Through Traffic (Vmax) from each node. Fig.7 presents this relationship, which exhibits a strong correlation.

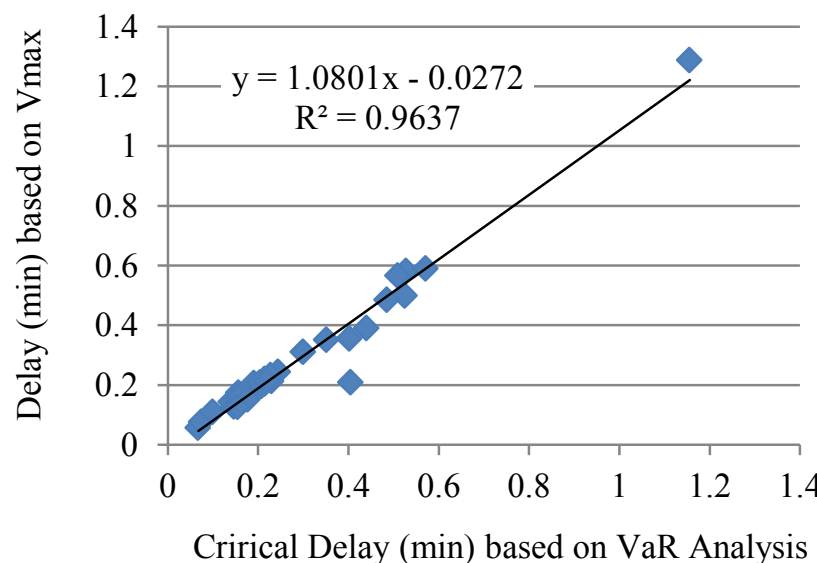

Fig. 7. Relationship between Critical Delay based on Vmax and VaR, Baseline

On the other hand, in Fig.8, no correlation is observed for the critical simulation step, where the critical delay is identified by the two different analyses (VaR analysis and the Maximum Through Traffic). By observing these two graphs it can be seen that (a) although the estimation of a critical delay can be similar in both analyses, (b) the identification of 
the critical time period can vary significantly, and it can lead the control strategy to be deployed in a different time period than the critical one.

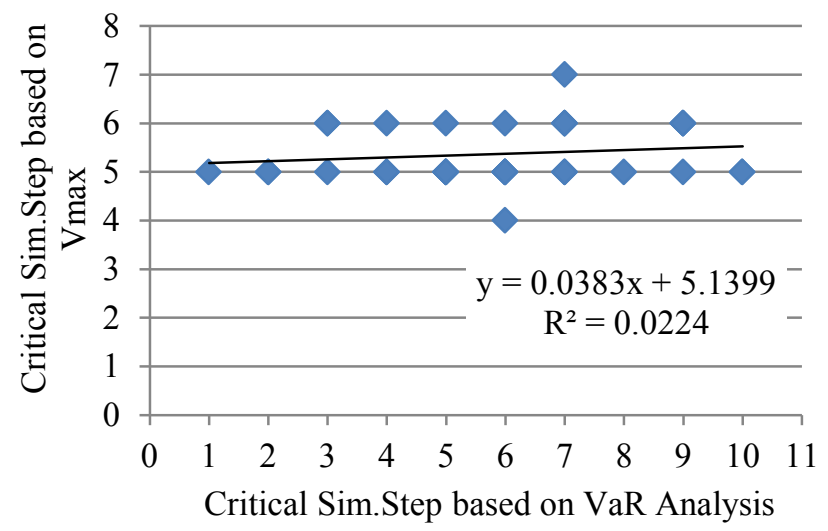

Fig. 8. Relationship between Critical Simulation Step based on Vmax and VaR, Baseline

A new traffic control strategy was formulated to manage demand. By identifying the 6th and the 7th simulation step as the critical steps, we deploy a new strategy 15 minutes prior to these simulation steps (i.e. proactively during the 5th and the 6 th simulation step), which adds $10 \%$ extra capacity at the main corridors of the network with direction towards the city centre. The increase in capacity represents an increase in effective green to the selected approaches and consequently a relative decrease to the opposing approaches. In this application this extension of green time ranges between 1.5 and 5 seconds. The new control strategy was formulated by minimizing $V a R$ in lieu of using conventional objective functions encompassing time attributes, such as delay. This strategy comprised all major coordinated corridors towards the city centre.

The same procedure (see Fig.1) was followed for evaluating the results from the new traffic control strategy to the network.

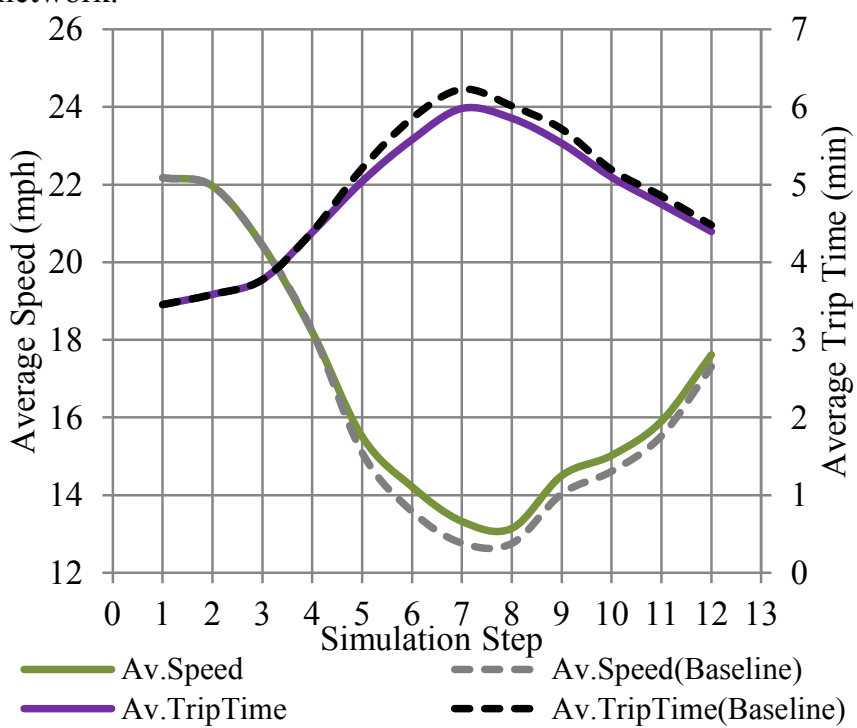

Fig. 9. Network statistics, New Strategy

The network statistics for the new control strategy are depicted in Fig.9, where it can be seen that the average speed during the critical time period is increased and consequently the average trip time is decreased. Fig.10 presents the histogram of the critical simulation steps with the new strategy. It can be seen that the critical simulation step are distributed more uniformly, even though the demand profile hasn't changed.

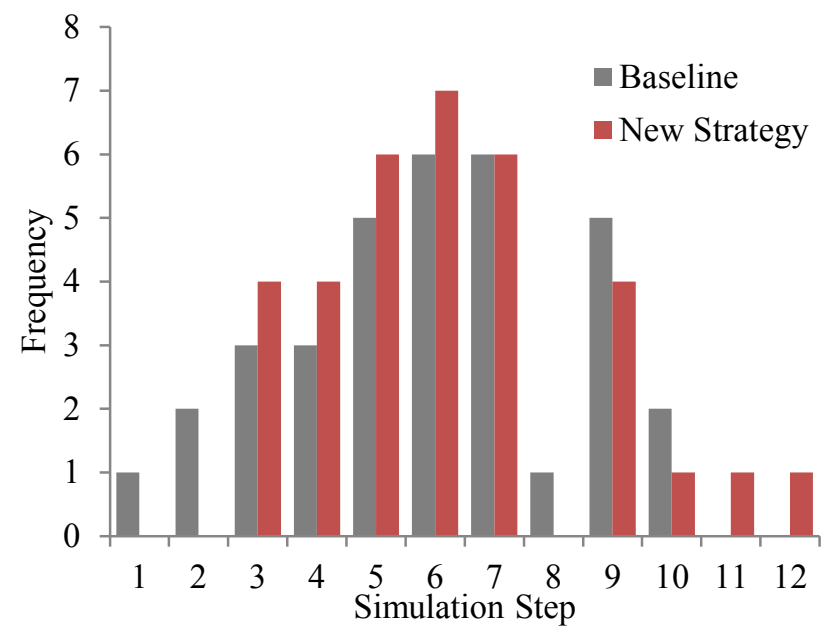

Fig. 10. Histogram of Critical Simulation Step, New Strategy

The comparison between the Critical Delay and the Simulation Step with regard to the $\mathrm{VaR}$ analysis and with regard to the Maximum Through Traffic from each node with the new strategy reveals the same correlation.

The overall savings in average delay in signalized nodes during the critical simulation step is $9 \%$, with $76 \%$ of the signalized nodes showing improvement in average delay during the critical simulation step. The improvement in total delay for all the signalized nodes (Fig.11) during the entire simulation horizon is $7.5 \%$. During each simulation step the decrease in total delay is ranging between $0 \%$ and $15 \%$.

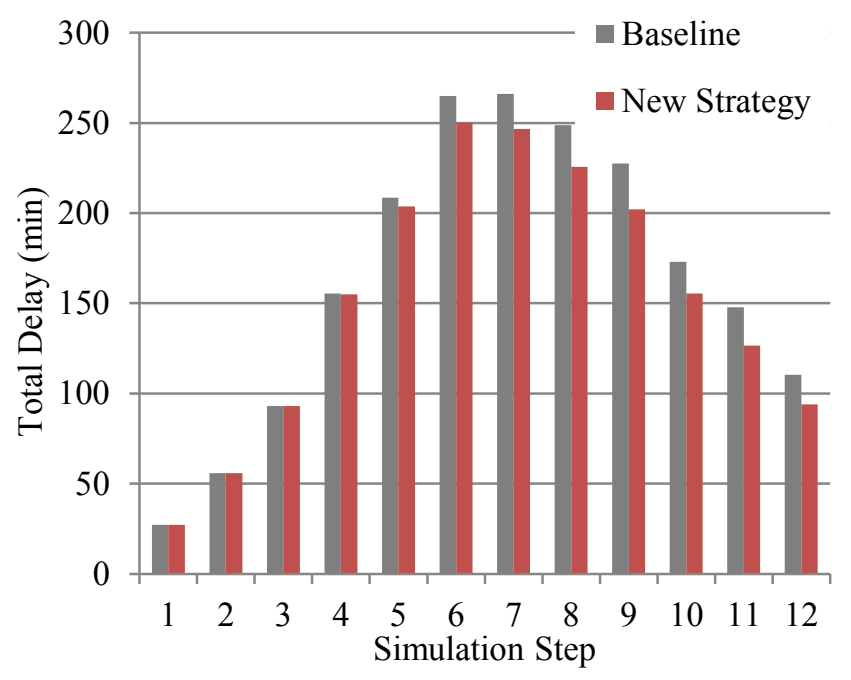

Fig. 11. Total Delay at signalised nodes, New Strategy

\section{CONCLUSIONS}

In this paper, we have formulated a methodology for testing different control strategies using dynamic traffic assignment tools applied at the City Centre of Gaborone (Botswana). The objective of our methodology is to assess the risk of the 
network efficiency resulting from the deployment of alternative traffic control strategies by incorporating the Value-at-Risk analysis, which is mainly used in financial applications. The proposed methodology is an iterative process where performance indices are calculated for each simulation step and their critical value and the corresponding simulation step is identified through the Value-at-Risk analysis and accessed in order to define the new traffic control strategy. Incorporating this methodology in real-time traffic control one can regard it as a process of Just-In-TimeSimulation, which can serve as a decision tool for combating peak-hour congestion. The computation time was measured on a dual-core PC running at $2.40 \mathrm{GHz}$ and with $3 \mathrm{~GB}$ installed memory and was equal to $40 \mathrm{sec}$.

The implementation of our methodology was conducted offline in the City of Gaborone, where by identifying the critical simulation steps, we deployed a control strategy which resulted in additional capacity in selected arterials in the direction towards the city centre. The computational experiments showed that the proposed analysis can provide valuable information regarding the time-dependent performance of the network and it can lead to a timedependent deployment of control adjustments based on the critical periods. Another benefit of the proposed methodology is that it can be easily implemented by researchers or practitioners to evaluate their alternatives and aid them to choose alternatives with less potential risks. The approach, as already mentioned, can be expanded to online procedures and deployment of control strategies to finer time resolution, i.e. 3-5 minutes.

\section{ACKNOWLEDGMENTS}

The authors wish to thank the Department of Roads, Ministry of Transport and Communications, Republic of Botswana for providing access to the database of the project 'Consultancy Services for Conceptual Design, Preparation of Bidding Documents, Assistance during the Selection of Contractor and Monitoring/Supervision of Construction, Installation, Operation and Maintenance for Traffic Signals Modernization and Provision of Centralized Traffic Control (CTC) for Greater Gaborone City'.

\section{REFERENCES}

Boyce, D, Lee, D.H. and Ran B. 'Analytical Models of the Dynamic Traffic Assignment Problem'. Networks and Spatial Economics, 1, 377-390, 2001.

Chang, G.L., Tao, X.D. 'An Integrated Model for Estimating Time-Varying Network Origin-Destination Distributions'. Transportation Research B, 22(3), 217232, 1999.

Cheliotis, G., Kenyon, C. 'Dynamics of Link Failure Events in Network Markets'. Netnomics, Vol.4, No.2, 163-185, 2002

DTALite. http://sourceforge.net/projects/dtalite/. Accessed October 2013.

Federal Highway Administration (FHWA), Sloboden, J., Lewis, J., Alexiadis, V., Chiu, Y.-C., Nava, E. 'Traffic Analysis Toolbox Volume XIV: Guidebook on the Utilization of Dynamic Traffic Assignment in
Modeling'. FHWA-HOP-13-015. FHWA, Washington D.C., 2012.

Mastroeni, L. and M. Naldi. 'Compensation Policies and Risk in Service Level Agreements: A Value-at-Risk Approach under the ON-OFF Service Model'. Economics of Converged, Internet-Based Networks, 6995, 2-13, 2011.

Merchant, D.K., Nemhauser, G.L. 'A Model and an Algorithm for the Dynamic Traffic Assignment Problem'. Transportation Science, 12, 183-199, 1978.

Peeta, S., Yang, T.-H. 'Stability issues for dynamic traffic assignment'. Automatica, 39, 21-34, 2003.

Peeta, S., and Ziliaskopoulos, A. 'Foundations of Dynamic Traffic Assignment: The Past, the Present and the Future'. Networks and Spatial Economics, 1, 233-265, 2001.

Robillard, P. 'Multipath Traffic Assignment with Dynamic Input Flows'. Transportation Research, 8, 567-573, 1974.

Shao, L. G., Qin, X. S., XuA, Y. 'Conditional Value-at-Risk Based Inexact Water Allocation Model'. Water Resources Management, 25, 9, 2125-2145, 2011.

Stathopoulos, A., Tsekeris, T., "A Hybrid Meta-Heuristic Optimization Algorithm for the Problem of O-D Matrix Estimation", Computer-Aided Civil and Infrastructure Engineering, Blackwell Publishing Journals, 2004.

Sun, H., Gao, Z. 'Robust Alpha-Reliable Network Design Problem under Distribution-free Demand'. Fifth International Joint Conference on Computational Sciences and Optimization, 453-457, 2012.

Tsekeris, T., Stathopoulos, A., 'Real-time dynamic O-D matrix adjustment using simulated and actual link flows in large-scale networks', $82^{\text {nd }}$ Annual Meeting of the Transportation Research Board, Washington, D.C., 2003.

Transportation Research Board (TRB), Chiu, Y.-C., Bottom, J., Mahut, M., Paz, A., Balakrishna, R., Waller, T., Hicks, J. 'Dynamic Traffic Assignment: A Primer'. Transportation Research Circular E-C153. Transportation Research Board, Transportation Network Modeling Committee, 2011.

Transportation Research Board (TRB). Highway Capacity Manual, Transportation Research Board National Research Council, Washington, D.C., USA, 2010.

Toumazis, I., Kwon, C., Batta, R. 'Value-at-Risk and Conditional Value-at-Risk Minimization for Hazardous Materials Routing. In: Handbook of OR/MS Models in Hazardous Materials Transportation, Springer, N.Y., 2013.

Wang, S., Watada, J., Pedrycz, W. 'Value-at-Risk-Based Two-Stage Fuzzy Facility Location Problems' IEEE TRANSACTIONS ON INDUSTRIAL INFORMATICS, VOL. 5, NO. 4, 2009.

Yagar, S. 'Dynamic Traffic Assignment by Individual Path Minimization and Queuing'. Transportation Research, 5, 179-196, 1971.

Ziliaskopoulos, A., Waller, S., Li, Y., Byram, M. 'LargeScale Dynamic Traffic Assignment: Implementation Issues and Computational Analysis'. Journal of Transportation Engineering, 130(5), 585-593, 2004. 